\section{AB1103 SEVERE PULMONARY ARTERIAL HYPERTENSION AS THE INITIAL MANIFESTATION OF SYSTEMIC LUPUS ERYTHEMATOSUS IN A 7-YEAR-OLD MALE PATIENT: A CASE REPORT}

M.T. Brana ${ }^{1,1}$, S. Osorio ${ }^{2}$, L. Aparicio ${ }^{2}$, A. Rodriguez ${ }^{1}$, E. Faugier ${ }^{2}$, R. Maldonado ${ }^{2}$. ${ }^{1}$ Pediatric Rheumatology; ${ }^{2}$ Hospital Infantil de Mexico, Federico Gomez, Hospital Infantil de Mexico Federico Gomez, Mexico, Mexico

Background: Pleural pulmonary manifestations in patients with systemic lupus erythematosus are reported in approximately $5 \%$ of cases. Presenting as pleural effusion, alveolar haemorrhage, diffuse interstitial lung disease, pulmonary infections and pulmonary arterial hypertension, among others, they are a manifestation difficult to diagnosis. Pulmonary arterial hypertension is a rare condition, usually occurring 3 to 5 years after the diagnosis of systemic lupus erythematosus.

Objectives: We report the case of a 7-year-old male patient who presented with pulmonary arterial hypertension as the initial manifestation of systemic lupus erythematosus.

Methods: Case report

Results: A 7-year-old male patient who was admitted to the Pneumology Department at Children's Hospital of Mexico Federico Gómez due to respiratory distress. In emergency assessment, pulmonary arterial hypertension of $66 \mathrm{mmHg}$ was identified, of unknown cause. The patient did not have significant medical background, having enjoyed of good health up to 6 months prior to his admission. He presented with a history of non-quantified fever, as well as episodes of fatigue and dyspnea. Two months before admission, chest pain was added, exacerbated with inspiration. On admission, transthoracic echocardiography revealed severe dilatation of right cavities, moderate tricuspid insufficiency, with left ventricular ejection fraction of $56 \%$ and arterial pulmonary pressure of $66 \mathrm{mmHg}$. The diagnostic approach is initiated. Due to a history of pulmonary tuberculosis in the patient grandmother, the patient was studied with BAAR and cervical lymph node biopsy, ruling out the diagnosis. Infectious process causing the manifestations was also ruled out. The patient was discharged with medical treatment, requiring readmission in for 7 days, with facial oedema and in lower extremities, generalised pallor, asthenia, adynamia and 4 days before a decrease in urinary volumes and frequency. On admission, right heart failure, secondary to increase of pulmonary hypertension for discontinuation of diuretic administration. A renal biopsy was performed, which was reported as class IV lupus nephropathy, with an index of activity and chronicity of 0 . The diagnosis of systemic lupus erythematosus is integrated based on the ACR criteria. Induction of remission of lupus nephropathy based on the CARRA protocol. As treatment was administered the patient showed important clinical improvement.

Conclusions: Pulmonary arterial hypertension is a rare condition, usually occurring 3 to 5 years after the diagnosis of systemic lupus erythematosus. In paediatric population, it is reported as a lupus complication in $5 \%$ to $14 \%$ of patients, and less than $1 \%$ as an initial manifestation. It is a clinical complication that gives the patient a high risk of morbidity and mortality. It is important to acknowledge that pulmonary arterial hypertension can be the initial manifestation of lupus in paediatric population. A prompt identification assures a prompt treatment and a better prognosis.

\section{REFERENCE:}

[1] Prete M1, Fatone MC, Vacca A, Racanelli V, Perosa F. Severe pulmonary hypertension as the initial manifestation of systemic lupus erythematosus: a case report and review of the literature. 2014 Mar-Apr;32(2):267-74. Epub 2013 Dec 16.

Disclosure of Interest: None declared DOI: 10.1136/annrheumdis-2018-eular.1883

\begin{tabular}{|l|l}
\hline AB1104 & MALIGNANCY-RELATED ARTHROPATHIES IN \\
PAEDIATRIC PATIENTS SEEN IN A TERTIARY \\
HOSPITAL
\end{tabular}

M.A. Bayo-Ang, C.B. Bernal, M.T.M. Collante. Pediatric Rheumatology, University of Santo Tomas Hospital, Manila, Philippines/Philippine Rheumatology Association, Manila, Philippines

Background: Along with constitutional symptoms such as fever, fatigue, and weight loss, childhood malignant diseases often also present with various musculoskeletal symptoms and findings, prompting consult or referral to a paediatric rheumatology clinic.

Objectives: The study described the characteristics of patients seen at the paediatric rheumatology clinic who were eventually diagnosed with malignancy.

Methods: Medical records of 35 children diagnosed with hematologic or solid malignancy who were seen at the paediatric rheumatology clinic from August 2013 to December 2017 because of with joint pains or difficulty in ambulation were reviewed.
Results: Twenty of the 35 patients $(57.1 \%)$ were males and 15 (42.9\%) were females. The mean age at diagnosis was 7.3 years old \pm 4.1 (range $2.5-17.0$ ). The time of diagnosis from the onset of symptoms has a mean of 4.5 months \pm 7.4 . Fever was the most common constitutional symptom (82.8\%), followed by pallor $(45.7 \%)$ and weight loss $(37.1 \%)$, while the most common musculoskeletal symp tom or finding was joint pains $(100 \%)$, followed by difficulty in ambulation $(40 \%)$, joint swelling (37.1\%) and nocturnal pains (31.4\%). Mean haemoglobin level was $93.9 \mathrm{~g} / \mathrm{L} \pm 18.9$. Mean white cell count was $11.9 \times 10^{9} / \mathrm{L} \pm 7.1$, mean segmenters was $41.6 \% \pm 21.7$, and mean lymphocytes was $53.0 \% \pm 24.2$. Mean platelet count was $294.0 \times 10^{9} / \mathrm{L} \pm 220.5$. Mean ESR was $99.6 \mathrm{~m} / \mathrm{h} \pm 33.7$ at baseline. Radiographic evaluation done were all normal. Peripheral blood smears and bone marrow aspiration study were done in all patients. Thirty-three patients (94.2\%) were diagnosed with acute leukaemia, 18 of which was acute lymphocytic leukaemia, 14 acute lymphocytic leukaemia pre-B, and 1 acute myelogenous. One patient had Non-Hodgkins Lymphoma and 1 patient had hemoblastoma.

Conclusions: Children with joint pains associated with findings atypical of a rheumatologic disorder presentation should be further investigated for the possibility of malignancy.

\section{REFERENCE:}

[1] Papp ZE et al; Early symptoms of childhood malignancy; Orv Hetil. 2017 May;158(21):829-834

Disclosure of Interest: None declared DOI: 10.1136/annrheumdis-2018-eular.4364

\section{AB1105 INFLUENCE OF JUVENILE IDIOPATHIC ARTHRITIS ON THE QUALITY OF LIFE OF YOUNG ADULTS IN THE TRANSITION PERIOD TO ADULT RHEUMATOLOGIC CARE IN UKRAINE}

M. Dzhus ${ }^{1}$, Y. Boyko ${ }^{2} .{ }^{1}$ Internal Medicine; ${ }^{2}$ O. Bohomolets National Medical University, Kyiv, ${ }^{2}$ Clinical Immunology, LVIV Medical University, Lviv, Ukraine

Background: Young adults with juvenile idiopathic arthritis (JIA) often have an active disease with significant functional impairment in adulthood that can affect their physical and mental functions.

Objectives: The aim of the study was to determine the impact of JIA on quality of life, mental and physical health in young adults with JIA during the transition healthcare in Ukraine.

Methods: The cross-sectional study of 89 young adults aged 16 to 22 years with a history of JIA and 25 age- and sex-matched controls (without rheumatic disease) were included. The study was performed at the Aleksandrivsky Central Clinical Hospital, Kyiv, Ukraine in the period between April 2015 and February 2017. There was performed an evaluation of the disease activity by JADAS, received therapy, quality of life by the SF36, the functional activity (HAQ), long-term JIA damage indices JADI-A and JADI-E, ${ }^{1}$ PHQ-9, Beck depression scale.

Results: Patients with JIA had worse $(p<0.001)$ physical health in comparison with the control group. Physical $(p<0.001)$ and role $(p<0.05)$ functioning and bodily pain $(p<0.001)$ in JIA patients were decreased, compared with the controls. However, the indicators responsible for psychological function in patients with JIA did not differ from the controls. The analysis of functional activity revealed a strong negative effect of the disease on physical role functioning $(p<0.001)$, bodily pain $(p<0.001)$, general health $(p<0.001)$, vitality $(p<0.001)$, social role functioning $(p<0.001)$, and mental health $(p<0.001)$, which are included in physical $(p<0.001)$ and mental $(p<0.05)$ health. HAQ had strong negative effects on physical functioning $(r=-0.56, p<0.001)$, role function $(r=-0.33, p<0.001)$, bodily pain $(r=-0,60$ $p<0.001)$, general health $(r=-0,40, p=0.01)$, vitality $(r=-0,46, p<0.001)$, social functioning $(r=-0.48, p<0.001)$ and mental health $(r=-0.42, p<0.001)$. Articular long term damages (JADI-A) have a predominantly negative effect on the patient's physical health $(r=-0,27, p<0,05)$ and on the indices associated with it: physical functioning $(r=-0,24, p<0.05)$, bodily pain $(r=-0.24, p<0.05)$, general health $(r=-0.24, p<0.05)$, vitality $(r=-0.19, p<0.05)$, social functioning $(r=-0.27, p<0.05)$, mental health $(r=-0.22, p<0.05)$. While the extra-articular long-term damages JADI-E also have a predominantly negative effect on the patient's physical health $(r=-0.22, p<0.05)$ and on the indexes associated with it: physical functioning $(r=-$ $0,28, p<0,05)$, bodily pain $(r=-0,20, p<0,05)$, general health $(r=-0,23, p<0,05)$, mental health $(r=-0.23, p<0.05)$, as well as a positive association with Beck depression scale $(r=0,28, p<0.05)$ and $P H Q-9(r=0.28, p<0.05)$.

Conclusions: In our transitional cohort of Ukrainian patients at the era of biological therapies, juvenile idiopathic arthritis had a larger effect on the physical than mental SF-36 subscale. Pain was the main factor influencing quality of life. Extraarticular long-term JIA damages have impact on physical and mental health of young adults. Additional evaluation of mental health by PHQ-9 and Beck depression scale is recommended for evaluation signs of depression in Ukrainian young adults in transition period. 